\title{
A família do adolescente com comportamento autolesivo internado no serviço de urgência de um hospital pediátrico. Estudo de caracterização sociodemográfica
}

La familia del adolescente con conducta autolesiva ingresado en el servicio de urgencias de un hospital pediátrico. Estudio de caracterización sociodemográfica

Families of Teenage Children with Self-injurious Behaviour Admitted to the Emergency Service in a Paediatric Hospital. Social-demographic Characterisation Study

\author{
Maria Edite Trinco ${ }^{1}$, José Carlos Santos ${ }^{2}$ \\ 'Enfermeira Especialista em Saúde Infantil e Pediátrica. Hospital Pediátrico. Coimbra, Portugal; Enfermeira Forense; Doutoranda em Enfermagem; \\ Assistente Convidada da Escola Superior de Enfermagem De Coimbra. Portugal. \\ 2Professor coordenador na Escola Superior de Enfermagem de Coimbra, Portugal. Doutorado em Saúde Mental.. \\ Contacto: edite.trinco@hotmail.com \\ Fecha de recepción: 16 de julio de 2018 / Fecha de aceptación: 2 de noviembre de 2018
}

\begin{abstract}
Resumo
A família é considerada a instituição mais antiga da Humanidade, que tem sido sujeita a múltiplas transformações devido ao advento de vários contextos culturais, sociais e económicos que se têm vivido, encontrando-nos assim perante uma constante renovação de paradigma, porém e apesar de todas as alterações a que esta tem sido exposta, a família enquanto instituição tem-se mantido como uma estrutura essencial em qualquer sociedade.

Objetivo(s): caracterização sociodemográfica da família dos adolescentes com comportamento autolesivo internados num hospital pediátrico da região centro.

Metodologia: estudo qualitativo, de natureza descritiva com recurso a análise de conteúdo.

Resultados: O perfil sociodemográfico da família é o da família nuclear, com uma média de 3,9 indivíduos por agregado familiar, os entrevistados têm uma média de idade de 43,5 anos, com um nível de escolaridade médio e vivem em meio rural, sendo o distrito de Coimbra o mais representado com $73,3 \%$, da amostra.

Conclusões: apesar de todas as alterações na estrutura familiar, esta continua a ter um papel primordial no apoio e acompanhamento dos adolescentes internados com comportamentos autolesivos, enfatizando a necessidade de uma visão sistémica, para a promoção de adaptações e mudanças necessárias ao desenvolvimento do adolescente e de todo o sistema familiar.
\end{abstract}

Palavras-Chave: família, adolescente, comportamento autodestrutivo.

\section{Resumen}

La familia se considera la institución más antigua de la humanidad. Objeto de múltiples cambios como consecuencia de los diversos contextos culturales, sociales y económicos vividos, nos encontramos ante una constante renovación del paradigma. Sin embargo, pese a todos los cambios a los que se ha visto expuesta, la familia, como institución, se ha mantenido como una estructura fundamental en cualquier sociedad. Objetivos: caracterización sociodemográfica de la familia de los adolescentes con conducta autolesiva ingresados en un hospital pediátrico de la región centro de Portugal. Metodología: estudio cualitativo, de tipo descriptivo a través del análisis de contenido. Resultados: el perfil sociodemográfico de la familia es el de una familia nuclear, con una media de 3,9 miembros por unidad familiar. Los encuestados tienen una media de edad de 43,5 años, un nivel educativo medio y viven en un entorno rural; el distrito de Coímbra es el más representado, con un $73,3 \%$ de la muestra. Conclusiones: pese a todos los cambios que ha sufrido la estructura familiar, esta sigue desempeñando una función primordial en el apoyo y en el acompañamiento de adolescentes ingresados por conducta autolesiva, y pone de manifiesto la necesidad de un enfoque sistémico con el fin de impulsar las adaptaciones y los cambios necesarios para el desarrollo del adolescente y de todo el sistema familiar.

Palabras clave: familia, adolescente, conducta autodestructiva. 


\begin{abstract}
The family is considered to be the oldest institution of Humanity, which has undergone many changes due to multiple cultural, social and economic contexts arising. It has thus been subject to ongoing paradigm shifts, yet, in spite of such changes, the family as an institution has remained the core structure of any society. Objective(s): Socialdemographic characterisation of the families of teenage children with self-injurious behaviour admitted to a paediatric hospital in the centre region. Methodology: Qualitative study, descriptive in nature, based on content analysis. Results: The social-demographic profile of the family is the nuclear family which consists in average of 3.9 people per household. Mean age of respondents is 43.5 years, with an average level of education and they live in the countryside. The district of Coimbra has the highest level of representation, accounting for $73.3 \%$ of the sample. Conclusions: In spite of all of the changes in the family structure, the latter still plays an essential role in supporting and accompanying teenagers with self-injurious behaviour admitted to hospital, emphasising the need for a systemic outlook for promoting the necessary adjustments and changes to the teenager's development and the entire family system.
\end{abstract}

Keywords: family, teenager, self-destructive behaviour.

\section{Introdução}

A família é considerada a instituição mais antiga da Humanidade, mas o seu conceito não se tem mantido constante ao longo dos tempos, o que tem dificultado a sua definição, podendo ser considerado um conceito subjetivo devido ao advento de múltiplos contextos culturais, sociais e económicos que se têm vivido desde a Idade Média, encontrando-nos assim perante uma constante renovação de paradigma ${ }^{1}$.

As mudanças de paradigma social foram-se instalando paulatinamente desde a Revolução Industrial. Esta mudança na estrutura familiar teve o seu apogeu com a saída da mulher para o mercado de trabalho no final do seculo XIX, com implicações significativas na redução da taxa de natalidade, que por sua vez teve implicações diretas na taxa de crescimento populacional, originando diferentes configurações familiares ${ }^{2}$. Então, o conceito é alargado e passa a ser definido como um sistema constituído por dois ou mais indivíduos, que têm um compromisso entre si, partilhando objetos e tomadas de decisões e onde os seus membros são autodefinidos ${ }^{3}$.

Devido ao progresso da investigação científica muitas outras definições têm surgido, eco de uma sociedade em constante mutação. Assim, define a família como sendo "um grupo de seres humanos vistos como uma unidade social ou um todo coletivo, composta por membros ligados através da consanguinidade, afinidade emocional ou parentesco legal, incluindo pessoas que são importantes para ocliente ",

Apesar das numerosas definições que tentam explanar o conceito de família e de todas as alterações a que esta tem sido exposta, a família enquanto instituição tem-se mantido como a base essencial de toda a sociedade, com uma responsabilidade relevante na realização, desenvolvimento e preservação da personalidade humana, incorporando o mais importante apoio na socialização e desenvolvimento dos seus membros, onde as condições das relações interpessoais e as exigências internas e externas necessitam de uma constante plasticidade para uma resposta assertiva em novos contextos e resolução de crises, não colocando em risco a sua sucessão, congruência, função e identidade e onde o todo é maior que a soma das partes.

Estas adaptações devem estar em harmonia com a fase de desenvolvimento da família e o ciclo vital da família, que vai desde a formação do casal, a família com filhos pequenos, com filhos na escola, com filhos adolescentes e a família com filhos adultos ${ }^{5}$, sendo cada etapa vivida pela família de forma individualizada, onde o subsistema familiar desempenha as suas tarefas de acordo com a fase que está a viver, proporcionando um desenvolvimento harmonioso e sem sobreposição de papéis e tarefas ${ }^{6}$. Mas é na fase dos filhos adolescentes que a família vive uma das maiores transições do seu ciclo, uma vez que esta fase do ciclo vital é vivida pela família como um dos períodos de maior vulnerabilidade ${ }^{7}$.

Sendo a família um agregado especialmente destinado à realização pessoal dos seus elementos tem como um dos papéis principais a socialização, educação, prestação de cuidados globais e transmissão de valores a todos os seus elementos, com especial relevância aos filhos, mantendo-se como base fundamental de apoio quando estes entram na adolescência, ajudando-os a constituir o seu próprio self, servindo de 
mentora na evolução e conhecimento, facilitando-lhes um crescimento harmonioso e em sincronia que conduza à sua autonomia e socialização responsáveis ${ }^{8}$.

A adolescência é uma etapa da vida que não reúne consenso na comunidade científica no que concerne ao seu início e fim. Não obstante, a Organização Mundial de Saúde ${ }^{9}$ dá-nos uma definição segundo a qual esta etapa da vida é compreendida entre os 10 e os 19 anos. Por seu turno a Ordem dos Enfermeiros ${ }^{10}$ considera-a uma fase decisiva para a adoção de hábitos de vida saudáveis, ou, pelo contrário, adversos que ponham em risco a sua saúde e bem-estar.

Esta etapa da vida do ser humano é talvez a de maior exigência adaptativa ao longo do seu ciclo vital, pois requer do adolescente um permanente ajuste devido a alterações físicas, hormonais, sociais, psicológicas e relacionais. Todavia, a maioria dos adolescentes vivencia esta transição de maneira ajustada e sem grandes tumultos, com as necessárias adaptações para uma vida saudável. Mas outros há que têm algumas dificuldades de ajuste e reajuste à mudança inerente a esta fase, colocando em risco um desenvolvimento holístico harmonioso, tornando-o vulnerável a comportamentos de risco, nomeadamente a comportamentos autolesivos $^{11}$.

Os comportamentos autolesivos são considerados pela comunidade científica como um problema de saúde pública necessitado de investigação, avaliação, prevenção e intervenção. $\mathrm{O}$ adolescente que tem um comportamento autolesivo vivencia um sofrimento psíquico de grande intensidade, vislumbrando no ato autolesivo o alívio para o sofrimento vivenciado ${ }^{12}$.

De acordo com o Plano Nacional de Prevenção do Suicídio $^{13}$, os comportamentos autolesivos podem envolver atos com ou sem intencionalidade de morte, e que são caracterizados por: ingestão de fármacos em doses superiores às doses terapêuticas, ingestão de uma droga ilícita ou substância psicoativa com o propósito de autoagressão, automutilação, ingestão de uma substância ou objeto não ingerível, saltar de um lugar relativamente elevado.

É na adolescência que os comportamentos autolesivos têm a sua iniciação, sendo mais frequentes entre as raparigas, com uma média de idade de 15 anos, sendo o modus operandi mais frequente a automutilação e/ou a ingestão de fármacos em doses superiores às doses terapêuticas $^{11,12}$. Por vezes estes comportamentos são recorrentes, pelo que a letalidade do método assim como a intencionalidade não devem ser menosprezadas, sobretudo porque nem sempre o adolescente tem uma real compreensão da perigosidade do ato ${ }^{11}$.

Um estudo de ${ }^{12}$ refere que a família de um adolescente que tenha tido um comportamento autolesivo vivencia momentos de grande sofrimento psíquico, com repercussões a nível familiar nesta fase de transição do ciclo vital da família, onde os seus membros estão mais vulneráveis e expostos a múltiplas adversidades, contudo com capacidade de adaptação às respetivas mudanças ${ }^{14}$.

Após uma pesquisa alargada na base de dados científicas publicadas, constatamos que não existe produção científica divulgada, sobretudo pela enfermagem, que caracterize a família do adolescente com comportamento autolesivo, pelo que nos parece pertinente a nossa investigação que visa essa caracterização, com enfoque na obtenção de conhecimento que leve à aquisição de novos instrumentos para as melhores práticas de enfermagem e consecutivo progresso científico.

Perante tais considerações delineámos como objetivo: conhecer o perfil sociodemográfico da família dos adolescentes com comportamento autolesivo que foram internados no serviço de urgência de um hospital pediátrico no período de janeiro a novembro de 2015 .

\section{Metodologia}

Para a consecução deste objetivo e tendo em conta as características da investigação, optámos por um estudo de cariz qualitativo, descritivo e exploratório, sendo os dados obtidos através de entrevistas semiestruturadas ao pai/mãe que acompanharam o adolescente no internamento, ficando os achados registados e codificados para efeitos de organização, apresentação e análise, utilizando a metodologia estatística com base na análise descritiva dos mesmos, com apoio do programa informático Excel, após autorização da comissão de ética da referida instituição com a Ref ${ }^{\mathrm{a}}$ CHUC-110-13.

\section{População e amostra}

Os participantes foram selecionados por meio de uma amostra de conveniência para que os resultados fossem profícuos e determinados no sentido de melhor servirem o nosso propósito. A recolha dos dados foi realizada no serviço de urgência de um hospital pediátrico da região centro do país, num período compreendido 
entre janeiro e novembro de 2015.

A amostra é constituída por trinta e quatro mães e quatro pais, cujos filhos adolescentes com idade compreendida entre os 13 e os 18 anos que tiveram um comportamento autolesivo e que ficaram internados no serviço de urgência de um hospital pediátrico da região centro.

\section{Critérios de inclusão e exclusão}

Trata-se de uma amostra sequencial, que inclui os pais cujo filho adolescente teve um comportamento autolesivo e que ficou internado no serviço de urgência, acompanhado pelo pai/mãe, e que aceitaram de forma livre e esclarecida participar na investigação.

Foram tidos como critérios de exclusão outro acompanhante que não fosse pai/mãe, pai/mãe de adolescente com comportamento autolesivo suicidário e família com perturbação psíquica em crise.

\section{Procedimento de colheita de dados}

Após parecer favorável da comissão de ética da referida instituição, com a Ref ${ }^{\mathrm{a}}$ CHUC-11013, a abordagem aos participantes foi feita aquando da primeira consulta após a alta do serviço de urgência, tendo sido explicado o propósito do estudo, garantido o anonimato e confidencialidade em concordância com o Código Deontológico que rege a profissão de enfermagem.

Os dados foram colhidos na consulta externa do serviço de pedopsiquiatria do hospital da área de residência da família, através de uma entrevista semiestruturada ao pai/mãe que acompanhou o adolescente durante o internamento, com recurso à gravação áudio, em ambiente adequado e previamente designado para o mesmo.

As entrevistas tiveram por base um guião elaborado pelos investigadores e que consistia na caracterização sociodemográfica da família do adolescente, através de uma pergunta aberta onde constava o género, a idade, a tipologia e agregado familiar, a escolaridade e proveniência da amostra. A duração média das entrevistas foi de 60 minutos, a todas foi atribuído um número que foi tomado como referência aquando da sua transcrição.

\section{Resultados}

Os dados foram trabalhados através da análise de conteúdo, que de acordo com deve ser alicerçada em três fases diferenciadas: a pré-análise, a análise dos dados; e o tratamento dos resultados. Para uma melhor compreensão dos dados, os mesmos serão apresentados sobre a forma de quadros síntese da análise efetuada às entrevistas.

Numa primeira abordagem apresentamos os dados segundo o género no Quadro 1, onde pode ser verificada a predominância do género feminino relativamente ao género masculino.

A idade dos pais é apresentada no Quadro2, com uma variação entre os 35 e os 65 anos de idade.

A tipologia familiar mais comum da nossa amostra é a da família nuclear, conforme o Quadro 3.

O agregado familiar varia entre as 2 e as 6 pessoas, sendo o mais persistente o de 4 pessoas, como se pode verificar no Quadro 4.

No Quadro 5, apresentamos a variação na escolaridade, sendo o mais comum o ensino secundário em ambos os progenitores.

Sendo a seleção feita no serviço de urgência de um hospital pediátrico da região centro e como hospital de referência na área da pediatria, a sua área de abrangência é de toda a região centro do país, como podemos verificar no Quadro 6, embora mais comum no distrito de Coimbra.

\section{Discussão}

Para melhor compreendermos o desenvolvimento individual precisamos de o contextualizar no espaço familiar, com todas as suas características individuais e organizativas e dinâmica própria.

Da análise dos dados podemos inferir que são as mães quem mais acompanha os filhos ao hospital e quem fica no internamento, totalizando cerca de $89,5 \%$ do total dos acompanhantes, sendo esta inferência corroborada pelos estudos de18,19 que nos dizem que são as mães quem mais cuida dos filhos.

No que respeita à idade dos pais, esta apresenta uma média de 43,5 anos, sendo que $71 \%$ tinham uma idade 
superior aos 42 anos. Também num estudo20 as idades dos participantes variam entre os 37 e os 58 anos.

Relativamente à composição familiar, $22(57,8 \%)$ são famílias nucleares, uma família adotante (2,6\%), 5 famílias alargadas (13,2\%), 5 famílias monoparentais $(13,2 \%)$ e 5 famílias reconstruídas $(13,2 \%$.), o que vai ao encontro dos dados publicados pelo Instituto Nacional de Estatística21.

Como já foi referido anteriormente, as configurações familiares têm sido alvo de grandes e profundas alterações no que concerne à sua estrutura. Com o advento de novas configurações familiares, o tipo de família nuclear deixou de ser o modelo de referência, contudo mantém-se o mais atual e presente na nossa sociedade. Em consonância com o Instituto Nacional de Estatística, cada vez mais nos deparamos com famílias recompostas, mas também com um crescimento significativo de famílias monoparentais, que se deve ao aumento do número de divórcios, mas também à ascensão da quantidade de mulheres que adotam filhos sozinhas, ou que por opção são mães solteiras.

Dos nossos resultados podemos ressalvar que a família do adolescente com comportamento autolesivo internado no serviço de urgência é constituída por uma média de 3,9 de indivíduos, sendo o agregado familiar mais comum constituído por 4 pessoas, maioritariamente pai, mãe e dois filhos.

Quanto à escolaridade dos pais, 4 (10,5\%) têm licenciatura, $26(68,5 \%)$ frequentaram o ensino secundário e $8(21 \%)$ têm a escolaridade básica.

Relativamente ao local de residência, $26(68,4 \%)$ famílias vivem em zona rural, e 12 (31,6\%) famílias vivem em meio urbano. A maioria das famílias $(73,8 \%)$ são do distrito de Coimbra, não podendo olvidar que se trata do distrito que acolhe o hospital onde se desenvolveu o estudo, com especial relevância para o facto de 55,3\% viverem em zona rural.

Estes resultados permitem constatar que os comportamentos autolesivos na adolescência são transversais no que respeita à sua complexidade, independentemente da configuração familiar, local de residência ou escolaridade dos pais, porque sendo a família considerada um sistema vivo que acolhe fenómenos aleatórios, consentindo inseguranças e oscilações no seu percurso, é primordial para a enfermagem a compreensão dos seus membros em geral e cada um em particular, tendo presente as suas vivências, necessidades, dificuldades, frustrações e desencantos, numa perspetiva de grupo que não pode ser negligenciado enquanto sistema, estando muitas vezes carente de compreensão e cuidados na sua integralidade.

\section{Conclusões}

De acordo com a análise e discussão dos resultados deste estudo, verifica-se que o perfil sociodemográfico da família do adolescente com comportamento autolesivo que recorre ao serviço de urgência de um hospital pediátrico da região centro de Portugal é o da família nuclear, cujos pais têm uma média de idade de 43,5 anos, com um nível de escolaridade médio, com uma média de 4 pessoas por agregado familiar e vivem em meio rural.

Compete pois aos técnicos de saúde respeitar a diversidade socioeconómica, cultural, espiritual, linguística, entre outras, das famílias, constituindo-as como parceiros dos cuidados, reconhecendo e promovendo o desenvolvimento das suas capacidades e restituindo-lhes o poder de decisão e de intervenção.

Independentemente das características das famílias, esta deve ser atendida com base numa visão sistémica da mesma, com o desígnio de contribuir para a promoção de mudanças estruturais de forma a ajudá-las na procura da melhor solução e adaptação à problemática em causa, demonstrando respeito e empatia por todos os seus membros, apoiando-as na tomada de decisão, ajudando-as nos momentos de crise, promovendo o seu crescimento, desenvolvimento e estratégias de empoderamento que contribuam para uma saúde familiar positiva e resolução de crise interna e/ou externa.

\section{Implicações para a Prática Clínica}

Esta investigação contribui com novos conhecimentos sobre a caracterização da família do adolescente com comportamento autolesivo, contribuindo para delinear cuidados centrados na família do adolescente com comportamento autolesivo de forma sistémica, sendo esta abordagem dos cuidados fundamental para uma melhor compreensão e intervenção de enfermagem.

\section{Bibliografia}

1. Relvas A, Alarcão M. Novas formas de família. Quarteto: Coimbra; 2002.

2. Sampaio $D$, Cruz $H$, \& Carvalho, $M$. Crianças e jovens em risco - $A$ família no centro da intervenção. (Princípia, Ed.) Fundação 
Calouste Gulbenkian: Cascais; 2011.

3.Hanson S. Enfermagem de cuidados de saúde à família - teoria,

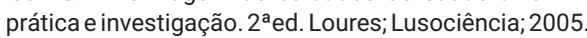

4. Ordem dos Enfermeiros (OE) Classificação Internacional para a Prática de Enfermagem (CIPE) 2010 Disponível em:http://associacaoamigosdagrandeidade.com/wpcontent/u ploads/filebase/guiasmanuais/ORDEM\%20ENFERMEIROS\%20 cipe.pdf. Consultado em 2 de março de 2018.

5. Alarcão M. (Des) equilíbrios familiares uma visão sistémica. Coimbra: Quarteto; 2006.

6. Figueiredo M. H. J. S. Enfermagem de família: um contexto do cuidar. Porto. Instituto de Ciências Biomédicas Abel Salazar.2009. Dis ponível em: ht tps://repositorio-

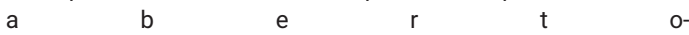
.up.pt/bitstream/10216/20569/2/Enfermagem\%20de\%20Famli a\%20Um\%20Contexto\%20do\%20CuidarMaria\%20Henriqueta $\% 2$ OFigueiredo.pdf. Consultado em 2 de março de 2018.

7.Relvas A. P. O ciclo vital da família: perspectiva sistémica (3a ed.). Porto: Edições Afrontamento; 2004.

8.Silveira P G, Wagner A. Ninho cheio: a permanência do adulto jovem em sua família de origem. Estudos de Psicologia, Campinas, v. 23, n. 4, p. 441453 , dez; 2006.

9. World Heatlh Organization (WHO) Adolescent health.2011. Disponível em: http://apps.who.int/adolescent/second-decade/. Consultado em 3 de abril de 2018.

10.Ordem dos Enfermeiros (OE) Guias orientadores da boa prática em enfermagem de saúde infantil e pediátrica. Lisboa, Portugal; 2010.

11. Trinco M E, Santos J C, Barbosa A. Vivências e necessidades dos pais no internamento do filho adolescente com comportamento autolesivo. Revista de Enfermagem Referência Série IV - n. ${ }^{\circ} 13$ ABR./MAI./JUN.pp. 115-124. ISSNe: 2182.2883 | ISSNp: 0874.0283; 2017.
12. Trinco M.E, Santos J C. O adolescente com comportamento autolesivo sem intenção suicida no internamento do serviço de urgência de um hospital pediátrico da região centro. Revista Portuguesa de Saúde Mental (Spe.5), 63-68; 2017.

13.Portugal. Plano Nacional de Prevenção do Suicídio 2013/2017, Direção G e ral da S aúde. Disponível e m: http://www.portaldasaude.pt/NR/rdonlyres/BCA196AB-74F4472B-B21E6386D4C7A9CB/0/i018789.pdf. Consultado em 3 de abril de 2018.

14. Zetterqvist, M. Child and Adolescent Psychiatry and Mental Health. 9:31 doi: 10.1186/s13034-015-0062-7; 2015.

15.Santos N, Neves E. Adolescência e comportamentos suicidários. Suicídio e comportamentos autolesivos. Dos conceitos à prática clínica. Ed. Carlos Saraiva, Bessa Peixoto \& Daniel Sampaio Lideledições técnicas, Lda., 2014. ISBN: 9789897520426; 2014.

16. Meleis A. I. Transitions theory. In M. C., Smith \& Parker, M. E., Parker (2015). Nursing theories \& nursing practice (4th ed), pp. 361-380. Philadelphia, PA: F. A. Davis Company; 2015.

17.Bardin L. Análise de conteúdo. Lisboa, Portugal: Almedina; 2009.

18. Lindgren B. M, Astrom S, Graneheim, U H. Held to ransom: Parents of self-harming adults describe their lived experience of professional care and caregivers. International Journal of Qualitative Studies on Health and Wellbeing, 5(3), 1-10. doi:10.3402/qhw.v5i3.5482; 2010.

19. Daly P. Mothers living with suicidal adolescents: A phenomenological study of their experiences. Journal of Psychosocial Nursing \& Mental Health Services, 43 (3), 22; 2005.

20. Russell S N. Experiences of Parents of Self-Harming Adolescent Children (Doctoral dissertation, Walden University); 2017.

21. Instituto Nacional de Estatística, I.P. Famílias nos Censos - Diversidade e Mudança. (Delgado A, Wall K, Edits.) Lisboa: Edição conjunta do Instituto Nacional de Estatística e da Imprensa de Ciências Sociais; 2011.

\begin{tabular}{|c|c|c|}
\hline Amostra & $\mathbf{n}$ & $\mathbf{\%}$ \\
\hline Mãe & 34 & 89,5 \\
\hline Pai & 4 & 10,5 \\
\hline Total & 38 & 100 \\
\hline
\end{tabular}

Quadro 1: Caracterização da amostra segundo o género

\begin{tabular}{|c|c|c|c|}
\hline Idade & $\mathbf{n}$ & $\mathbf{\%}$ & \multirow{2}{*}{ Média global } \\
\cline { 1 - 3 } $35-40$ & 10 & 26,3 & \multirow{2}{*}{43,5} \\
\cline { 1 - 3 } $41-50$ & 20 & 52,6 & \\
\hline $51-65$ & 8 & 21,1 & \\
\hline Total & 38 & 100 & \\
\hline
\end{tabular}

Quadro 2: Caracterização da amostra segundo a idade 


\begin{tabular}{|c|c|c|}
\hline Tipologia & $\mathbf{n}$ & $\mathbf{\%}$ \\
\hline Nuclear & 22 & 57,8 \\
\hline Reconstruída & 5 & 13,2 \\
\hline Monoparental & 5 & 13,2 \\
\hline Alargada & 5 & 13,2 \\
\hline Adotiva & 1 & 2,6 \\
\hline Total & 38 & 100 \\
\hline
\end{tabular}

Quadro 3: Caracterização da tipologia familiar

\begin{tabular}{|c|c|c|}
\hline Agregado familiar & $\mathbf{n}$ & $\mathbf{\%}$ \\
\hline 2 pessoas & 3 & 7,9 \\
\hline 3 pessoas & 11 & 28,9 \\
\hline 4 pessoas & 16 & 42,1 \\
\hline 5 pessoas & 6 & 15,8 \\
\hline 6 pessoas & 2 & 5,3 \\
\hline Total & 38 & $100 \%$ \\
\hline
\end{tabular}

Quadro 4: Caracterização do agregado familiar

\begin{tabular}{|c|c|c|c|c|c|}
\hline Escolaridade & $\begin{array}{c}\mathbf{n} \\
\text { mãe }\end{array}$ & $\mathbf{\%}$ & $\begin{array}{c}\mathbf{n} \\
\text { pai }\end{array}$ & $\mathbf{\%}$ & $\begin{array}{c}\mathbf{\%} \\
\text { total }\end{array}$ \\
\hline Ensino básico & 7 & 18,5 & 1 & 2,6 & 21,1 \\
\hline Ensino secundário & 24 & 63,2 & 2 & 5,2 & 68,4 \\
\hline Ensino superior & 3 & 7,9 & 1 & 2,6 & 10,5 \\
\hline Total & 34 & 89,6 & 4 & 10,4 & 100 \\
\hline
\end{tabular}

Quadro 5: Caracterização da escolaridade

\begin{tabular}{|c|c|c|c|c|c|}
\hline Local de residência & $\begin{array}{c}\mathbf{n} \\
\text { rural }\end{array}$ & $\mathbf{\%}$ & $\begin{array}{c}\mathbf{n} \\
\text { urbano }\end{array}$ & $\mathbf{\%}$ & $\begin{array}{c}\mathbf{\%} \\
\text { total }\end{array}$ \\
\hline Coimbra & 21 & 55,3 & 7 & 18,4 & 73,7 \\
\hline Guarda & 2 & 5,3 & 1 & 2,6 & 7,9 \\
\hline Aveiro & 3 & 7,9 & 1 & 2,6 & 10,5 \\
\hline Leiria & 2 & 5,3 & 1 & 2,6 & 7,9 \\
\hline Total & 28 & 73,8 & 10 & 26,2 & 100 \\
\hline
\end{tabular}

Quadro 6: Caracterização da residência 